\title{
Developing and evaluating interventions to reduce inappropriate prescribing by general practitioners of antibiotics for upper respiratory tract infections: A randomised controlled trial to compare paper-based and web-based modelling experiments
}

\author{
Shaun Treweek ${ }^{1 *}$, lan W Ricketts ${ }^{2}$, Jillian Francis ${ }^{3}$, Martin Eccles ${ }^{4}$, Debbie Bonetti ${ }^{5}$, Nigel B Pitts ${ }^{5}$, \\ Graeme MacLennan ${ }^{3}$, Frank Sullivan ${ }^{1}$, Claire Jones ${ }^{2}$, Mark Weal ${ }^{6}$, Karen Barnett ${ }^{1}$
}

\begin{abstract}
Background: Much implementation research is focused on full-scale trials with little evidence of preceding modelling work. The Medical Research Council Framework for developing and evaluating complex interventions has argued for more and better theoretical and exploratory work prior to a trial as a means of improving intervention development. Intervention modelling experiments (IMEs) are a way of exploring and refining an intervention before moving to a full-scale trial. They do this by delivering key elements of the intervention in a simulation that approximates clinical practice by, for example, presenting general practitioners (GPs) with a clinical scenario about making a treatment decision.
\end{abstract}

Methods: The current proposal will run a full, web-based IME involving 250 GPs that will advance the methodology of IMEs by directly comparing results with an earlier paper-based IME. Moreover, the web-based IME will evaluate an intervention that can be put into a full-scale trial that aims to reduce antibiotic prescribing for upper respiratory tract infections in primary care. The study will also include a trial of email versus postal invitations to participate.

Discussion: More effective behaviour change interventions are needed and this study will develop one such intervention and a system to model and test future interventions. This system will be applicable to any situation in the National Health Service where behaviour needs to be modified, including interventions aimed directly at the public.

Trial registration: ClinicalTrials (NCT): NCT01206738

\section{Background}

Although much is known about interventions that can be effective in changing the behaviour of health professionals [1], the literature provides little information to guide the choice, or to optimise the components, of these interventions $[2,3]$. Implementation research is the scientific study of methods to promote the uptake of

\footnotetext{
* Correspondence: streweek@mac.com

'Quality, Safety and Informatics Research Group, University of Dundee, Kirsty Semple Way, Dundee, UK

Full list of author information is available at the end of the article

research findings to improve the quality of care. However, much implementation research is focused on fullscale trials with little evidence of preceding modelling work. The Medical Research Council (MRC) framework for developing and evaluating complex interventions has argued for more and better theoretical and exploratory work prior to a trial as a means of improving intervention development [4].

The use of intervention modelling experiments (IMEs) for interventions that aim to change behaviour is one approach to doing exploratory work [5]. In an IME key 
elements of the intervention are delivered (generally in a randomised controlled trial, hence use of the word 'experiment') in a manner that approximates the real world but the measured outcome is generally an interim outcome, a proxy for the clinical behaviour of interest (clinical decision in response to a simulated clinical encounter) prior to entering the intervention into a fullscale trial. To date IMEs have been paper-based $[5,6]$ but this may limit their efficiency, acceptability and ecological validity. Web-based IMEs have the potential to provide much richer simulations of clinical encounters and allow measurement of key process variables such as time to make a decision. The current study will develop a web-based IME system based around an existing webbased intervention delivery system called Lifeguide http://www.lifeguideonline.org/[7]. We will then use the new system to run a web-based IME on antibiotic prescribing for upper respiratory tract infections (URTIs) in general practice.

Cochrane reviews of the effectiveness of antibiotics in treating URTIs suggest no benefit in colds [8] and only marginal benefit for uncomplicated sore throat [9]. A large cohort study of the use of antibiotics for respiratory tract infection in UK primary care concluded that antibiotics are not justified to reduce the risk of serious complications in URTIs [10]. Primary care accounts for $80 \%$ of total antimicrobial use and $60 \%$ of that is for respiratory infections, which are on the whole self-limiting [11]. The web-based IME will systematically develop and evaluate theory-based interventions for reducing unnecessary prescribing of antibiotics that correspond to the theoretical, modelling and experimental phases of the MRC framework. We will directly compare this work with a previously published paper-based IME on the same topic [6], allowing us to evaluate the two methods of developing and simulating interventions prior to a full-scale trial. We propose that the most effective intervention will go on to be evaluated in a separately funded fullscale trial.

\section{Trial objectives}

1. To run a web-based IME to develop and evaluate theory-based interventions to reduce antibiotic prescribing for URTIs in primary care.

2. To compare the effectiveness of email and postal invitations to general practitioners (GPs) to take part in the web-based IME in a randomised controlled trial.

3. To compare the web-based and paper-based methods of running IMEs in terms of identification of predictors, the type of intervention that can be simulated, and the effect on intended prescribing behaviour on trial conduct of an IME.

\section{Methods}

Trial design

General

The study will include two trials, one a web-based IME, the other an evaluation of the method used to invite GPs to take part in the web-based IME. The basic design of these two trials will be:

1. Web-based IME: a three-arm, double-blind, randomised controlled trial of two behaviour change interventions compared to generic information.

2. Invitation: a two-arm, randomised controlled trial of an email invitation to GPs compared to a postal invitation.

The web-based IME will be based on a previous paper-based IME [6]. This earlier work provided qualitative data about the kind of attitudes and beliefs among GPs about the use of antibiotics in the treatment of URTIs (cough/cold, sore throat/tonsillitis in patients of any age). This was used to develop questionnaire items relevant to the clinical context of treating URTIs in primary care and which operationalised the constructs of our chosen theories (e.g., theory of planned behaviour: questionnaire has an item asking GPs whether they thought patients expected them to prescribe an antibiotic, which is linked to the theory's subjective norms construct). The earlier work also developed clinical scenarios describing patients presenting in primary care with symptoms of URTI. GPs were asked to report their clinical decisions in response to the scenarios and these were used to generate scores for 'simulated behaviour.' We will use a web-based IME to deliver the questionnaire and scenarios to GPs in order to identify predictors of prescribing behaviour and compare these with those identified in the paper-based IME.

The research study is based in the Division for Clinical and Population Sciences and Education at the University of Dundee at Ninewells Hospital, with support from the Universities of Aberdeen, Newcastle and Southampton and the Scottish School of Primary Care. It is funded by the Chief Scientist Office, grant number $\mathrm{CZH} / 4 / 610$ and approved by the Tayside Committee on Medical Research Ethics A, REC reference 10/S1401/54. A flow diagram of the study is shown in Figure 1.

\section{Participants}

Inclusion criterion is GPs in Scotland. Exclusion criterion is the inability to obtain both an email address and a postal address for the GP.

\section{Identifying participants}

GPs will be identified by the Scottish Primary Care Research Network (SPCRN; http://www.sspc.ac.uk/ $\operatorname{spcrn} /$ ) [12] using a combination of publicly available information provided by ISD Scotland http://www.isdscotland.org/isd/3793.html[13] and restricted information 


\section{Assessed for eligibility} (GPs in Scotland for whom we have both email and postal address) $(n=3000+)$

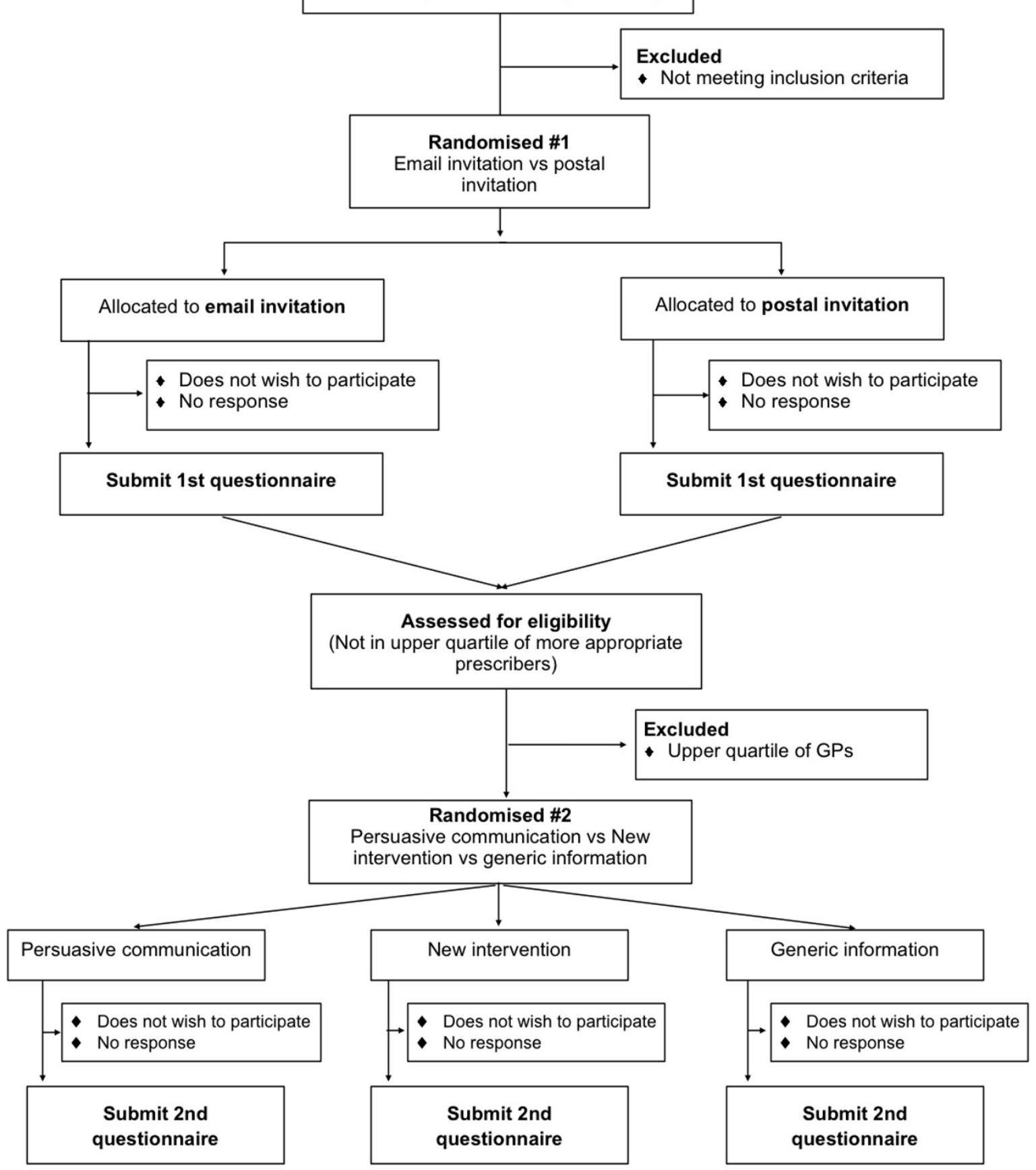

Figure 1 Flow diagram - Randomised controlled web-based IME with an embedded trial of email versus postal invitation to participate. 
held on the NHS.net database. A preliminary test of our recruitment method has identified over 3,000 GPs for whom we can obtain both email and postal addresses.

GPs receiving a postal invitation will receive a onepage letter and a two-page information sheet. Together with general information, the letter will contain a URL to the web-based IME system and a unique identifier for the GP. The identifier will be used to identify nonresponders and for payment (see below). GPs receiving an email invitation will receive an email containing the same text as on the paper letter and the two-page information sheet as a pdf attachment. We will send two reminders to non-responders, the first at two weeks, the second at four weeks, using the same method as used for the initial invitation. We expect response rates of $30 \%$ to $40 \%$ based on the earlier paper-based IME [6].

Participants will be asked to complete up to two online questionnaires, which together will form a webbased IME. GPs will be paid $£ 20$ in advance in the form of a voucher for the first part of the web-based IME and $£ 30$ in advance as a voucher for the second part. A recent Cochrane review of strategies to increase response rates to postal and electronic questionnaires found that advanced payment led to a higher response rate odds ratio $=2.00 ; 95 \%$ confidence interval $=1.54$ to 2.60) [14]. GPs will be able to select from a list of seven types of voucher: Amazon, Argos, Boots, iTunes, Love2Shop, Marks \& Spencer, or Starbucks. GPs will also be free to do the study without receiving a voucher if they so wish.

GPs will be able to decline to take part in the study by clicking a button on the second page of the web-based IME system, by emailing their ID number to a study email address, or by telephoning or sending a text message to the study mobile number. A flow diagram of the study is shown in Figure 1.

\section{Informed consent}

GPs will not be expected to provide explicit consent; a completed questionnaire will be taken as implied consent.

\section{Randomisation}

The study statistician will generate a list of random numbers to be used by SPCRN to randomly allocate GPs to receive either an email or postal invitation on a 1:1 basis. There will be no stratification. The list will be generated using Stata 11.0 statistical software (StataCorp, College Station TX). To ensure we do not approach too many GPs to participate in the main study we will randomise two initial blocks of 250 GPs (see Sample size) then subsequent blocks of 50 GPs will be contacted until the required sample size for the webbased IME is met.

All GPs will complete the same, initial web-based questionnaire to identify predictors of behavioural intention and simulated behaviour. GPs will receive one of two interventions (one of which will be a persuasive communication) or a comparator with the second webbased questionnaire. The study statistician will generate a list of random numbers for allocation to intervention or comparator on a 1:1:1 basis, which will be loaded into the web-based IME system. Allocation will, therefore, be done by the system when the GP logs onto the web-based IME system to complete the second questionnaire. There will be no stratification.

\section{Blinding}

Study participants, the trialists, and the trial statistician will be blind to allocation. Staff at the SPCRN will know the allocation for the email versus postal invitation study but not the web-based IME intervention study.

\section{Trial procedures}

Participants will be asked to complete up to two online questionnaires, which together will form a web-based IME. GPs who are already likely to be following best evidence with regard to prescribing antibiotics will be excluded from the second questionnaire, which we will do by excluding those in the first quartile of 'intention to prescribe' responses to the first questionnaire. These $25 \%$ of GPs will be thanked but will not continue in the study. Thus $75 \%$ of GPs completing the first questionnaire will be asked to complete the second. The intervention will be delivered together with the second questionnaire.

\section{Duration}

Participants will remain in the study until they complete the second questionnaire, which we expect to be between four and five months after they receive the invitation to take part.

\section{Interventions and comparator Interventions}

The web-based IME will deliver one of the interventions used in the paper-based IME [6] - a persuasive communication - and a second, new intervention developed for the web-based IME. The persuasive intervention aimed to reinforce the GP's beliefs about the positive consequences of managing sore throat without prescribing antibiotics. The materials for the persuasive communication can be downloaded at http://www.biomedcentral. com/content/supplementary/1472-6963-8-11-s2.doc[15]. This intervention did change GPs' beliefs in the paperbased IME, and we aim to see if this is replicated in the web-based IME.

However, we will also deliver a new intervention that takes advantage of the possibilities of web delivery. This will allow a comparison between an intervention simulated using web and paper-based methods and, additionally, provide a head-to-head comparison between that intervention and an intervention developed without the constraints of paper delivery. 
The new intervention, designed to take advantage of the possibilities offered by web-based delivery, will be designed using predictors identified from the first questionnaire and by using appropriate behaviour change techniques $[16,17]$. There is no widely agreed taxonomy by which to specify methods of delivery of behaviour change techniques; we will use as a starting point the suggestions of the Evidence-based Behavioural Medicine Committee (EBMC) [18]. Suggested dimensions are: provider, format, setting, recipient, duration, and frequency. We will use the EBMC suggestions to map the effectiveness of the interventions in the primary studies included in the systematic reviews populating it with the delivery methods used in the studies in the systematic reviews of strategies to decrease antibiotic prescribing $[19,20]$ and the results from the first questionnaire. We will send the results of the mapping exercise to a small number of experts for external validation.

We will use 'intervention mapping' [21] to systematically consider combinations of content and methods of delivery; there are likely to be logical combinations. By considering such combinations we will produce a range of potential new interventions.

We will pilot each of the potential new interventions (up to three) with groups of three to six GPs. Whilst delivering the intervention we will ask them to 'think aloud' about their reactions to the intervention, which allows us to identify problems and fine-tune content and delivery. We will select the most promising intervention for evaluation in the web-based IME. GPs involved in developing the intervention will not be included in the web-based IME.

\section{Comparator}

The comparator will be provision of general information about prescribing of antibiotics taken from recent reviews on antibiotic prescribing $[8,9]$.

\section{Outcome measures}

For the web-based IME, the primary outcomes will be behavioural intention and behavioural simulation, which have been shown to be reliably related to behaviour $[16,22]$. Three questions will assess GPs' strength of intention to manage URTIs without antibiotics (e.g., over the next 12 weeks, when a patient presents with a URTI, I have in mind to manage them without prescribing an antibiotic (rated on 7-point Likert scales 'Strongly Disagree' - 'Strongly Agree'). Responses will be summed with a low score corresponding to a low intention to prescribe antibiotics. Sixteen clinical scenarios from the paper-based IME work will provide the materials to measure simulated behaviour (eight in the first questionnaire, eight more in the second). Respondents will be asked how they will manage the patients in the scenarios and asked to rate, on a score out of 10, the difficulty of making their clinical decision.
Secondary outcomes are concerned with evaluating the relative utility of web-based and paper-based IMEs. For this, we will compare the following outcomes for each method.

1. Behaviour change techniques that can be operationalised We will develop a matrix of behaviour change techniques that can be operationalised by each IME method. This work will be done with researchers (especially health psychologists) unconnected with the project in brainstorming workshops. Project team members will present work done using paper-based and web-based IMEs, and participants will work alone to place behaviour change techniques into the matrix. Following this group discussion will be used to reach consensus on which techniques could be supported by each method. We will do this with more than one group of researchers (e.g., at weekly departmental research meetings at collaborator sites). Here we are looking for the method that can be to deliver most behaviour change techniques.

\section{Complexity of scenarios that can be delivered We} will develop a table of factors (e.g., people involved, context) that we are able to vary in clinical scenarios that can be delivered by each IME method. We will use brainstorming workshops involving researchers unconnected to the project to validate this list (i.e., do they agree with it) and make suggestions as to whether it can be extended (or reduced) for each IME method. We may use the same groups of researchers as for point one above although not at the same workshop. Here we are looking for the method that would allow more factors of a scenario to be varied.

3. Identification of predictors The first part of the webbased IME will evaluate whether the delivery mechanism of the IME (paper or web) affects predictors of GP behaviour. We anticipate that the predictors coming from the two methods will be similar because only the delivery method will be different; the web-based IME will use materials from the earlier paper-based IME. For example, evidence of habitual behaviour was strongly correlated with behavioural intention in the earlier paper-based IME. When delivering the same scenarios to GPs in a web-based IME, we anticipate (but do not know) that we will also find a strong correlation between habit and intended behaviour. Therefore, here we are seeking confirmation that delivery method alone does not lead to widely different predictors being identified by the IME.

4. Recruitment (reaching target and time taken to do so) Here, we will assess three things: time (in days) spent by the project team to recruit to target; time (in days) between sending an invitation to a potential participant and receiving a completed questionnaire; and the number of invitations necessary to receive one completed questionnaire 
These will be measured in the first part of the webbased IME and compared to estimates of timing (admittedly, less accurate) from the earlier paper-based IME. We will, however, get an indication of how the two methods compare. The embedded trial of paper invitation to GPs to participate in our study versus email invitation will provide comparative data on these two recruitment methods, which will be of wider relevance than recruitment to IMEs. Here we are looking for the fastest and least labour-intense method of recruiting participants to an IME.

5. Ability to change targeted constructs The theories used in paper- and web-based IMEs target particular constructs (e.g., GPs' beliefs about antibiotic prescribing) and the effect an intervention has on these targets is measured using a score. The web-based IME will involve a direct comparison of an intervention from the paperbased IME and a new intervention that makes use of the possibilities offered by web-based delivery. We will also compare the effect on targeted constructs of the same persuasive communication intervention delivered by both methods. By comparing each IME method's ability to change scores on the targeted constructs we will be able to make a statement as to which IME method is most effective.

\section{Effect size for persuasive communication interven-} tion compared to control The persuasive communication intervention and control were delivered in an earlier paper-based IME. By delivering the same intervention in a web-based IME we can compare the effect on the primary outcome (intention to prescribe an antibiotic) of the two delivery methods. Here, we are looking for the greatest effect size.

7. Qualitative work: ease of administration and GP feedback Running an IME is not trivial and a method that provides some administrative benefits (e.g., easier to put scenarios together, easier to pilot scenarios with some participants, easier to change the order of scenarios, easier to deliver to participants, easier to manage collected data) has efficiency advantages. We will compare, via interviews, experiences of the research team with running paper-based and web-based IMEs. This will, of course, be subjective (although item four will provide some quantitative information about effort linked to recruitment) but will nevertheless provide useful information about the effort required by researchers to run an IME via each approach. Here, we are looking for the method that is considered easiest to use and which uses fewest resources for an IME.

We will also consider qualitative feedback from GPs on the web-based delivery method and the utility of decision time data collected in the web-based IME, which is impossible to measure with paper-based IMEs. This work will also consider how the differing configurations of the interventions might explain their effects. Here, we are looking for feedback from GPs that identifies whether they think web-based IMEs have face validity, that the approach is feasible, and that they would participate in future web-based IMEs.

For the email versus postal invitation trial, the primary outcome is the number of GPs completing the first questionnaire. There are no secondary outcomes.

\section{Safety}

No safety issues or potential adverse events linked to participation are envisaged.

\section{Statistics}

\section{Sample size}

Our sample size will be 250 GPs completing the first questionnaire, $75 \%$ of whom will be invited to complete the second questionnaire. GPs who are already likely to be following best evidence with regard to prescribing antibiotics will be excluded from the second questionnaire, which we will do by excluding those in the first quartile of 'intention to prescribe' responses to the first questionnaire. These $25 \%$ of GPs will be thanked but will not continue in the study. For the first questionnaire analysis, our required sample size will be 162 based on a recommendation by Green [23] for multiple regression analysis with 14 predictor variables. The analysis of the questionnaire data will check the internal consistency of the measures and use multiple regression analysis to examine the relationships between predictor and outcome variables.

Using the dependent variable of behavioural intention, we will seek to detect an effect size of 0.66 . This was the mean effect size for change in intention in a metaanalysis of trials that measured change in intention and behaviour [24]. This 'medium to large' change in intention led to a 'small to medium' change in behaviour. We will require 50 participants per group to have $90 \%$ power of detecting this effect size at a significance level of $5 \%$ to 150 participants in total. We anticipate having 187 GPs in our $75 \%$ sample from the first questionnaire study, which will allow for some dropout.

The effect of each intervention will be estimated by comparing the change in intention in each of the active intervention groups with that in the control group. This procedure will be repeated with behavioural simulation as the dependent variable. We propose to take the intervention with the largest effect size forward into a definitive trial; there is a risk of wrongly choosing this as the most effective (a type 3 error). With 50 practitioners per group if treatment $B$ is actually superior to treatment A, such that the standardised difference in mean scores is 0.38 , the probability of incorrectly choosing treatment $\mathrm{A}$ is $5 \%$. 


\section{Statistical analysis}

For the web-based IME trial, reporting will be in line with the CONSORT statement [25]. Data will be summarised by intervention group using appropriate descriptive statistics where required, for example, mean and standard deviation for the primary outcomes. The primary outcomes will be analysed using an analysis of covariance (ANCOVA) framework to correct for the baseline levels of intention and behavioural simulation. Effect sizes will presented with estimated 95\% confidence intervals of the two new interventions versus generic information as the comparator. All analysis will be by intention to treat. Missing data at baseline are impossible by trial design, missing outcome data due to nonresponse to second questionnaire will not be imputed, analyses will be by responders only. Characteristics of responders and non-responders baseline data will be compared to assess the impact potential non-response on results. Secondary outcome will be analysed in a similar manner to the primary outcomes.

For the email versus postal invitation trial, reporting will also be in line with the CONSORT statement [25]. The primary outcome will be presented as the proportion of GPs sent an invitation completing the first questionnaire by invitation method. Proportions will be compared using a simple chi-square test, and results presented as an odds ratio and 95\% confidence interval. There are no secondary analyses for this trial.

\section{Discussion}

The National Health Service and other healthcare providers need effective quality improvement interventions to be put into clinical practice, which requires effective behaviour change interventions. IMEs are a way of exploring and refining an intervention before moving to a full-scale trial as recommended in the MRC framework for evaluating complex interventions. IMEs do this by delivering key elements of the intervention in a simulation that approximates clinical practice by, for example, presenting GPs with a clinical scenario about making a treatment decision. Earlier IMEs have been paper-based, which limits what can be done in the simulation.

Web-based IMEs provide the potential for better clinical simulations, which can be expected to lead to better interventions. The study described here will run a full, web-based IME involving 250 GPs that will advance the methodology of IMEs by directly comparing results with an earlier paper-based IME. Moreover, the web-based IME will evaluate an intervention that can be put into a full-scale trial that aims to reduce antibiotic prescribing in primary care. Reducing inappropriate prescribing of antibiotics in general practice is a national priority; indeed, antibiotic use is increasing in the UK and
Scotland's prescribing is second highest amongst UK administrations. More effective behaviour change interventions are needed, and this proposal will develop one such intervention and a system to model and test future interventions. This system will be applicable to any situation in a healthcare system where behaviour needs to be modified, including interventions aimed directly at the public.

We believe the web-based IME system will reduce the overall cost of trials by requiring fewer iterations of fullscale trial/analysis/revisions/full-scale trial before an optimised intervention is produced, thereby making better use of limited research budgets for trials. Finally, a web-based system can involve a wide range of stakeholders, expanding the range of opinions that can feed into intervention design and development, and enhancing the implementation potential of quality improvement initiatives.

\section{Acknowledgements and funding}

This study is funded by the Chief Scientist Office of Scotland, reference number $\mathrm{CZH} / 4 / 610$.

\section{Author details}

${ }^{1}$ Quality, Safety and Informatics Research Group, University of Dundee, Kirsty Semple Way, Dundee, UK. ${ }^{2}$ School of Computing, University of Dundee, Queen Mother Building, Dundee, UK. ${ }^{3}$ Health Services Research Unit, University of Aberdeen, Health Sciences Building, Foresthill, Aberdeen, UK. ${ }^{4}$ Institute of Health and Society, University of Newcastle, Baddiley-Clark Building, Richardson Road, Newcastle upon Tyne, UK. ${ }^{5}$ Clinical and Population Science and Education, University of Dundee, Kirsty Semple Way, Dundee, UK. ' ${ }^{6}$ chool of Electronics and Computer Science, University of Southampton, Southampton, UK.

\section{Authors' contributions}

All authors contributed to the design of the study. ST wrote the first draft of the protocol and all authors contributed to the final version. All authors have approved the final manuscript.

\section{Competing interests}

Martin Eccles is Co-Editor in Chief of Implementation Science; all decisions on this manuscript were made by another editor.

Received: 27 December 2010 Accepted: 3 March 2011

Published: 3 March 2011

\section{References}

1. Grimshaw JM, Shirran L, Thomas R, Mowatt G, Fraser C, Bero L, Grilli R, Harvey E, Oxman AD, O'Brien M: Changing provider behavior: An overview of systematic reviews of interventions. Medical Care 2001, 39(Supplement 2):॥2-1145.

2. Walker AE, Grimshaw J, Johnston M, Pitts N, Steen N, Eccles M: PRIME PRocess modelling in ImpleMEntation research: selecting a theoretical basis for interventions to change clinical practice. BMC Health Services Research 2003, 3:22.

3. Foy R, Eccles M, Jamtvedt G, Young J, Grimshaw J, Baker R: What do we know about how to do audit and feedback? BMC Health Services Research 2005, 5:50.

4. MRC: Developing and evaluating complex interventions: new guidance. 2008.

5. Bonetti D, Eccles M, Johnston M, Steen N, Grimshaw J, Baker R, Walker A, Pitts N: Guiding the design and selection of interventions to influence the implementation of evidence-based practice: an experimental simulation of a complex intervention trial. Social Science and Medicine 2005, 60:2135-2147. 
6. Hrisos S, Eccles M, Johnston M, Francis J, Kaner EF, Steen N, Grimshaw J: An intervention modelling experiment to change GPs' intentions to implement evidence-based practice: using theory-based interventions to promote GP management of upper respiratory tract infection without prescribing antibiotics \#2. BMC Health Services Research 2008, 8:10.

7. Joseph JA, Yardley L, Hare J, Osmond A, Yang Y, Weal M, Wills G: Application of the LifeGuide: The development and quantitative analysis of the 'Internet Doctor.'. Proceedings 5th International Conference on eSocial Science, Cologne 2009.

8. Arroll B, Kenealy T: Antibiotics for the common cold and acute purulent rhinitis. Cochrane Database of Systematic Reviews 2005, , 3: CD000247.

9. Del Mar CB, Glasziou PP, Spinks AB: Antiobiotics for sore throat. Cochrane Database of Systematic Reviews 2006, , 4: CD000023.

10. Petersen I, Johnson AM, Islam A, Duckworth G, Livermore DM, Hayward AC: Protective effect of antibiotics against serious complications of common respiratory tract infections: retrospective cohort study with the UK General Practice Research Database. BMJ 2007, 335:982-988.

11. Scottish Medicines Consortium, Scottish Antimicrobial Prescribing Group: Prudent Antimicrobial use in Primary Care - Respiratory (Paper 13). October meeting, Glasgow 2009.

12. Scottish Primary Care Research Network (SPCRN). [http://www.sspc.ac.uk/ spcrn/].

13. Information Services Division (ISD). [http://www.isdscotland.org/isd/3793. html].

14. Edwards PJ, Roberts I, Clarke MJ, Diguiseppi C, Wentz R, Kwan I, Cooper R, Felix LM, Pratap S: Methods to increase response to postal and electronic questionnaires. Cochrane Database of Systematic Reviews 2009, , 3: MR000008.

15. Biomedcentral. [http://www.biomedcentral.com/content/supplementary/ 1472-6963-8-11-s2.doc].

16. How do behaviour change techniques map on to psychological constructs?. Results of a consensus process. 19th Annual conference of the European Health Psychology Society; Galway, Ireland. Psychology and Health 2005.

17. Michie S, Johnston M, Abraham C, Lawton R, Parker D, Walker A: 'Psychological Theory' Group. Making psychological theory useful for implementing evidence based practice: a consensus approach. Quality and Safety in Health Care 2005, 14(1):26-33.

18. Davidson KW, Goldstein M, Kaplan RM, Kaufmann PG, Knatterud GL, Orleans CT, Spring B, Trudeau KJ, Whitlock EP: Evidence-based behavioral medicine: what is it and how do we achieve it? Annals of Behavioral Medicine 2003, 26(3):161-171.

19. Arnold SR, Straus SE: Interventions to improve antibiotic prescribing practices in ambulatory care. Cochrane Database of Systematic Reviews 2005, , 4: CD003539.

20. Ranji SR, Steinman MA, Shojania KG, Sundaram V, Lewis R, Arnold S, Gonzales R: Antibiotic prescribing behavior. In Closing the quality gap: a critical analysis of quality improvement strategies. Technical Review 9. Volume 4. Edited by: Shojania KG et al. Rockville MD: Agency for Healthcare Research and Quality; 2006:255-261.

21. Bartholomew LK, Parcel GS, Kok G: Intervention mapping: a process for developing theory- and evidence-based health education programs. Health Education and Behaviour 1998, 25(5):545-563.

22. Eccles MP, Johnston M, Hrisos S, Francis J, Grimshaw J, Steen N, Kaner EF: Translating clinicians' beliefs into implementation interventions (TRACII): a protocol for an intervention modelling experiment to change clinicians' intentions to implement evidence-based practice. Implementation Science 2007, 2(27):.

23. Green S: 'How many subjects does it take to do a regression analysis?'. Multivariate Behavioural Research 1991, 26:499-510.

24. Webb $T L$, Sheeran P: Does changing behavioural intention engender behaviour change? A meta-analysis of the experimental evidence. Psychological Bulletin 2006, 132(2):249-268.

25. Moher D, Hopewell S, Schulz KF, Montori V, Gøtzsche PC, Devereaux PJ, Elbourne D, Egger M, Altman DG: CONSORT 2010 Explanation and Elaboration: updated guidelines for reporting parallel group randomised trials. BMJ 2010, 340:c869. doi:10.1186/1748-5908-6-16

Cite this article as: Treweek et al:: Developing and evaluating interventions to reduce inappropriate prescribing by general practitioners of antibiotics for upper respiratory tract infections: A randomised controlled trial to compare paper-based and web-based modelling experiments. Implementation Science 2011 6:16.

\section{Submit your next manuscript to BioMed Central and take full advantage of:}

- Convenient online submission

- Thorough peer review

- No space constraints or color figure charges

- Immediate publication on acceptance

- Inclusion in PubMed, CAS, Scopus and Google Scholar

- Research which is freely available for redistribution

Submit your manuscript at www.biomedcentral.com/submit
Biomed Central 